\title{
Resultados de Técnicas de Reprodução Assistida em Pacientes Previamente Submetidas a Cirurgia Ovariana para o Tratamento da Endometriose
}

\author{
Outcome of Assisted Reproduction Treatment in Patients with Previous \\ Ovarian Surgery for Endometriosis \\ Selmo Geber ${ }^{1,2}$, Luis Felipe Víctor Spyer Prates ${ }^{2}$, Daniela Parreiras Ferreira ${ }^{2}$, \\ Liana Sales ${ }^{1}$, Marcos Sampaio ${ }^{1}$
}

\begin{abstract}
RESUMO
Objetivo: avaliar os efeitos da cirurgia ovariana prévia para o tratamento da endometriose na resposta ovariana durante ciclos de reprodução assistida e na taxa de gravidez subseqüente. Métodos: foram avaliadas 61 mulheres inférteis, com cirurgia ovariana prévia para endometriose, submetidas a 74 ciclos de fertilização in vitro/injeção intracitoplasmática de espermatozóides (FIV/ICSI) (Grupo caso), e comparadas a 74 pacientes inférteis, submetidas a 77 ciclos de FIV/ICSI no mesmo periodo e na mesma clínica, sem cirurgia ovariana prévia ou endometriose (Grupo controle). As pacientes foram pareadas por idade e tratamento realizado. A FIV foi realizada utilizando-se o protocolo longo para dessensibilização hipofisária seguida de indução da foliculogênese, monitorizada por ultra-sonografia e dosagem de estradiol. Após punção folicular os oócitos eram inseminados ou injetados e os embriões obtidos foram transferidos entre o dia 2 e dia 5 pós-inseminação.

Resultados: pacientes com menos de 35 anos previamente submetidas a cirurgia ovariana recrutaram número menor de oócitos quando comparadas às pacientes do grupo controle ( $p=0,049)$. O número de ampolas utilizadas, a duração da foliculogênese, o número de foliculos, a taxa de fertilização e de gravidez (53 e 56,2\%, respectivamente, para os grupos caso e controle) foram semelhantes. Mulheres com idade superior a 35 anos com cirurgia ovariana prévia necessitaram de maior número de ampolas para superovulação $(p=0,0017) e$ apresentaram um número menor de folículos e oócitos $(p=0,001)$. Um total de 10 pacientes ficaram grávidas no grupo caso (34,5\%) e 14 no grupo controle $(48,3 \%)(p=0,424)$.

Conclusão: a cirurgia ovariana para tratamento da endometriose reduz a resposta ovariana durante ciclos de FIV/ICSI em mulheres $>35$ anos e apresenta tendência a diminuir as taxas de gravidez. Assim, acreditamos que, para as pacientes inférteis com endometriose ovariana, o tratamento conservador deve ser considerado como primeira opção a fim de se evitar redução na resposta ovariana.
\end{abstract}

PALAVRAS-CHAVE: Infertilidade. Endometriose. Fertilização in vitro.

\section{Introdução}

A endometriose é entidade clínica caracterizada pela presença de tecido endometrial (glândulas e estroma) fora da cavidade uterina, com

\footnotetext{
${ }^{1}$ Origen - Centro de Medicina Reprodutiva

${ }^{2}$ Faculdade de Medicina da Universidade Federal de Minas Gerais

Correspondência: Selmo Geber

Av. Contorno 7747

30120-110 - Belo Horizonte - MG

e-mail: sjgeber@bhnet.com.br
}

atividade celular ${ }^{1}$. Sua incidência varia de 2 a $50 \%$, de acordo com o serviço, e as principais manifestações clínicas são a infertilidade e dor pélvica ${ }^{2-4}$. A sua associação com infertilidade é bem clara, especialmente quando há distorção anatômica e oclusão tubária, entretanto, até o momento, nenhum estudo demonstrou se a simples presença de pequenos focos endometrióticos ou endometriomas de pequenas dimensões prejudicariam a fertilidade.

Apesar de alguns autores terem tentado demonstrar associação entre infertilidade e endo- 
metriose sugerindo disfunções endócrinas ${ }^{5,6}$, outros mostraram falta de significância clínica ${ }^{7,8}$. Recentemente, foi proposta associação entre endometriose e disfunção imune que poderia prejudicar a fertilidade e a implantação ${ }^{9}$. Entretanto, essa associação ainda não está confirmada e, no momento, não há evidências conclusivas de que a endometriose, como fator isolado, seja causa de infertilidade.

Diferentes tipos de tratamento clínico e/ou cirúrgico têm sido propostos para a endometriose associada à infertilidade. A ablação cirúrgica ou remoção dos implantes tem sido usada há quase um século ${ }^{10}$, entretanto, não há evidências convincentes de que esta modalidade de tratamento promova melhora significativa da fertilidade ${ }^{11}$. Mais recentemente, Marcoux et al. ${ }^{12}$ demonstraram melhora significativa nas taxas de gravidez após ablação de implantes endometrióticos (endometriose minima) quando comparado ao grupo não tratado. Entretanto, a taxa de fecundação foi pequena quando comparada ao que era esperado para as chances habituais.

Em casos de endometriose extensa, associada a endometriomas, quando a cirurgia é indicada, é importante se considerarem os riscos do tratamento. Em pacientes inférteis é fundamental que, além de evitar a formação de aderên$\operatorname{cias}^{13}$, a função ovariana seja preservada. A remoção de endometriomas ou ablação de lesões endometrióticas sobre a superficie ovariana pode levar a uma redução da reserva folicular, com conseqüente piora da resposta à estimulação ${ }^{14-17}$. Além disso, em alguns casos, pode ser necessária a remoção de um ou dois ovários. Nesses casos, a cirurgia será mais nociva para o futuro reprodutivo da paciente que a própria endometriose. Assim, o objetivo do nosso estudo foi avaliar os efeitos da cirurgia ovariana prévia para o tratamento da endometriose na resposta ovariana à estimulação e nas taxas de gravidez, em ciclos de reprodução assistida.

\section{Pacientes e Métodos}

Efetuamos estudo retrospectivo no qual foram incluídas 66 mulheres com infertilidade primária, submetidas há mais de um ano à cirurgia ovariana (laparoscopia ou laparotomia) para retirada de cisto endometriótico (endometrioma). Foram excluídas da amostra inicial 5 pacientes $(3,3 \%)$ que desenvolveram falência ovariana prematura, tendo sido tratadas por meio de doação de oócitos. As 61 pacientes restantes com média de idade de 34,7 anos (DP 3,8 e variação de 23 a 43) foram submetidas a 74 ciclos de fertilização in vitro/ injeção intracitoplasmática de espermatozóides (FIV/ICSI) (Grupo caso) para tratamento de infertilidade na Clínica Origen - Centro de Medicina Reprodutiva - Belo Horizonte - Minas Gerais, no periodo de junho de 1995 a janeiro de 2001. As cirurgias ovarianas realizadas foram: ooforectomia unilateral ( $\mathrm{n}=14$ pacientes, 19 ciclos), ooforectomia unilateral com cistectomia contra-lateral $(n=1$ paciente, 1 ciclo) e cistectomia unilateral $(n=46$ pacientes, 54 ciclos). Outras 74 pacientes foram incluídas como grupo controle e apresentavam infertilidade primária. A média era de idade de 34,7 anos $( \pm 3,8$ com variação de 23 a 43). Estas não tinham cirurgia ovariana prévia nem indícios de endometriose e foram submetidas a $77 \mathrm{ci}$ clos de FIV/ICSI, no mesmo periodo de tempo, na mesma clínica. As pacientes foram pareadas de acordo com a idade e tratamento realizado, isto é, hormônios administrados para indução da superovulação, método de inseminação e dia da transferência dos embriões. As causas de infertilidade nas pacientes do grupo controle foram: fator tubário ( $\mathrm{n}=26$ pacientes, 26 ciclos), fator masculino ( $\mathrm{n}=21$ pacientes, 21 ciclos), infertilidade sem causa aparente ( $\mathrm{n}=15$ pacientes, 17 ciclos) e outros fatores ( $\mathrm{n}=12$ pacientes, 13 ciclos).

A comparação entre os grupos foi realizada de acordo com a faixa etária das pacientes. Desse modo, elas foram separadas em dois subgrupos: Grupo caso A (<35 anos) - 45 ciclos; Grupo caso B (>35 anos) - 29 ciclos; Grupo controle A ( $<35$ anos) - 48 ciclos; Grupo controle B (>35 anos) - 29 ciclos. As variáveis comparadas entre os grupos foram o número de ampolas de gonadotrofinas utilizadas para superovulação, duração da foliculogênese (dias), o número de folículos no dia da administração da gonadotrofina coriônica humana (hCG), o número de oócitos aspirados, a taxa de fertilização e a taxa de gravidez.

Todas as pacientes foram submetidas à propedêutica completa para infertilidade e apenas aquelas que apresentaram niveis séricos do hormônio folículo-estimulante (FSH) $<15 \mathrm{pg} / \mathrm{mL}$, no terceiro dia do ciclo menstrual, foram incluídas no estudo. Para os casais que apresentaram contagem de espermatozóides $<2,5 \times 10^{6}$ no dia da inseminação, foi realizada a ICSI.

O tratamento para indução da ovulação era iniciado com a administração subcutânea de 3,6 mg de análogo do hormônio liberador de gonadotrofinas (GnRHa - Goserelin - Zoladex Zeneca, Brasil) para supressão da função hipofisária (protocolo longo). Para a confirmação da dessensibilização hipofisária, foi realizada dosagem sérica de estradiol $\left(\mathrm{E}_{2}\right)$ e ultra-sonografia endovaginal aproximadamente 12 dias depois. Caso a concentração 
de estradiol $\left(\mathrm{E}_{2}\right)$ fosse menor que $30 \mathrm{pg} / \mathrm{mL}$ e a ultrasonografia mostrasse espessura endometrial menor que $3 \mathrm{~mm}$, as pacientes eram consideradas aptas para iniciar a indução da ovulação. Se elas ainda não estivessem prontas, o $\mathrm{E}_{2}$ sérico e o ultrasom eram repetidos a cada 2 dias até que a supressão fosse alcançada.

Após a confirmação da dessensibilização da função hipofisária, as pacientes eram submetidas à superovulação com doses diárias de gonadotrofinas humanas (hMG) ou injeções intramusculares de FSH recombinate ( $\mathrm{rFSH}$ Puregon - Organon, Brasil). A dose inicial de hMG ou rFSH era definida de acordo com a idade da paciente e ajustada de acordo com os niveis de $\mathrm{E}_{2}$. $\mathrm{O}$ crescimento folicular era monitorizado por meio de ultra-sonografia endovaginal (Tosbee - Toshiba, Japão). O hCG (10.000 UI - Pregnyl - Organon, Brasil) era administrado quando no mínimo dois folículos atingiam pelo menos $17 \mathrm{~mm}$ de diâmetro com niveis concordantes de $\mathrm{E}_{2}$.

A captação oocitária era realizada aproximadamente 34 horas após a administração do hCG por aspiração guiada por ultra-som vaginal. Os oócitos eram inseminados aproximadamente 5 horas depois (dia 0), tanto pela técnica clássica de fertilização in vitro (FIV) com aproximadamente 30.000 espermatozóides móveis/oócito, quanto por ICSI. O preparo do sêmen era feito pela técnica de swim up. A micromanipulação era realizada sobre uma placa aquecedora colocada em um microscópio invertido com aumento de 400 vezes (Nikon Diaphot, Japão), adaptado com um par de micromanipuladores hidráulicos e controle elétrico (Narishige, Japão). Um único espermatozóide era aspirado pela pipeta de injeção, a partir de uma gota de meio de cultura tamponado (Hepes-buffered Earle's balanced salt solution - Sigma, USA), contendo $10 \%$ de polivinilpirrolidona (PVP - Irvine, USA). Os oócitos eram mantidos em uma pipeta de sustentação enquanto a pipeta de injeção era empurrada através da zona pelúcida, para dentro do citoplasma, e um único espermatozóide era então injetado.

No dia seguinte, 17-19 horas após a inseminação (dia 1), os oócitos eram verificados para se confirmar a fertilização, pela presença de dois pró-núcleos. Os embriões foram cultivados em gotas de $20 \mu \mathrm{L}$ de solução balanceada de Earle (EBSS - Sigma) acrescida de $10 \%$ de soro sintético a $37^{\circ} \mathrm{C}$ em placa de Petri (Falcon - BD, USA), coberto com óleo mineral (Sigma), em mistura gasosa contendo $5 \%$ de $\mathrm{CO}_{2}{ }^{18}$. No dia 2 ou 3 após a captação oocitária, os embriões eram examinados e no máximo quatro eram selecionados para a tranferência, dependendo da idade da paciente. No caso da transferência ser realizada em estágio de blastocisto, os embriões eram transferidos para o meio S2
(Scandinavian IVF - Suécia) até o $5^{\circ}$ dia, quando um máximo de 3 blastocistos era transferido ${ }^{19}$.

O suporte de fase lútea era feito com progesterona vaginal (Utrogestan, França - 600 mg/ dia) por 12 dias. O nível sérico de hCG era dosado 12 dias após a tranferência dos embriões. A confirmação da gravidez era feita por meio de ultrasonografia endovaginal duas e quatro semanas depois. Todas as gravidezes foram seguidas por pelo menos 20 semanas.

$\mathrm{Na}$ análise estatística, foram comparados os dois grupos separados por faixas etárias e para isto utilizou-se o teste não paramétrico de KruskalWallis. Quanto à ocorrência ou não de gravidez foi utilizado o teste de Fisher. Os resultados são apresentados por média + desvio-padrão. Todos os resultados foram considerados significativos para uma probabilidade de significância inferior a $5 \%$ ( $p<0,05)$, tendo, portanto, pelo menos $95 \%$ de confiança nas conclusões apresentadas.

\section{Resultados}

A média de idade das pacientes do Grupo de estudo foi similar à do Grupo controle tanto para as com menos de 35 anos $(32,0 \pm 2,9$ e 32,1 12,8 anos, respectivamente), quanto para as com mais de 35 anos $(37,7 \pm 1,6$ e $37,9 \pm 1,8$ anos, respectivamente). Com relação às pacientes com idade $<35$ anos, não houve diferença estatística entre os grupos quanto ao número de ampolas utilizadas para a superovulação, na duração da foliculogênese (dias) e no número de folículos. As pacientes que tinham sido previamente submetidas a cirurgia ovariana apresentaram menor número de oócitos capturados em relação às pacientes do Grupo controle $(\mathrm{p}=0,049)$. A taxa de fertilização foi similar nos dois grupos (Tabela 1). O mesmo foi observado na taxa de gravidez (Figura 1), sendo que 24 pacientes $(53,3 \%)$ com cirurgia ovariana prévia e $27(56,2 \%)$ do grupo controle ficaram grávidas (diferença não significativa, $p=0,836$ ).

Tabela 1 - Resposta ovariana em pacientes com 35 anos ou menos e com cirurgia ovariana prévia comparadas ao grupo controle (sem cirurgia ovariana prévia) em ciclos de reprodução assistida.

\begin{tabular}{lccc}
\hline Variáveis & $\begin{array}{c}\text { Cirurgia ovariana } \\
\text { prévia }(\mathbf{n}=\mathbf{4 5})\end{array}$ & $\begin{array}{c}\text { Grupo controle } \\
(\mathbf{n}=\mathbf{4 8})\end{array}$ & $\mathbf{p}$ \\
\hline Ampolas & $39,1 \pm 8,6(26-60)$ & $39,4 \pm 9,5(12-56)$ & 0,501 \\
Foliculogênese (dias) & $11,1 \pm 1,5(8-14)$ & $11,2 \pm 1,9(8-14)$ & 0,496 \\
Folículos & $9,6 \pm 5,3(3-27)$ & $10,9 \pm 5,0(4-25)$ & 0,148 \\
Oócitos & $9,8 \pm 5,4(2-29)$ & $12,0 \pm 5,9(4-33)$ & 0,049 \\
Fertilização & $0,6 \pm 0,2(0,2-1)$ & $0,6 \pm 0,2(0,2-1)$ & 0,954 \\
Resultados: média \pm desvio padrão & & \\
(variação) & & &
\end{tabular}




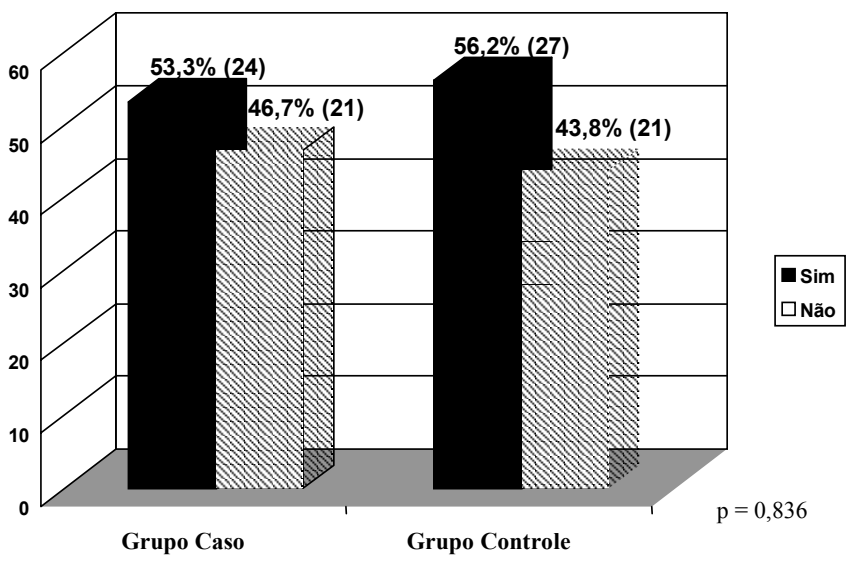

Figura 1 - Comparação entre as taxas de gestação após ciclos de reprodução assistida em pacientes $\leq 35$ anos com cirurgia ovariana prévia e no grupo controle.

No grupo de pacientes com mais de 35 anos, observamos que aquelas com cirurgia ovariana prévia necessitaram de maior número de ampolas para a indução da superovulação $(56,1$ contra 47,3 ; $\mathrm{p}=0,017)$ e apresentaram um menor número de folículos $(6,9$ e 10,6) e oócitos que as mulheres do grupo controle $(6,9$ e 11,9 respectivamente; $\mathrm{p}=0,001)$. A duração da foliculogênese foi similar nos dois grupos, assim como a taxa de fertilização (Tabela 2). Um total de 10 pacientes apresentou gravidez após o tratamento no grupo caso $(34,5 \%)$. No grupo controle, 14 mulheres $(48,3 \%)$ ficaram grávidas após o tratamento ( $\mathrm{p}=0,424)$ (Figura 2).

Tabela 2 - Resposta ovariana em pacientes com 35 anos com cirurgia ovariana prévia e grupo controle em ciclos de reprodução assistida.

\begin{tabular}{lccc}
\hline Variáveis & $\begin{array}{c}\text { Cirurgia ovariana } \\
\text { prévia }(\mathbf{n}=\mathbf{2 9})\end{array}$ & $\begin{array}{c}\text { Grupo controle } \\
(\mathbf{n}=\mathbf{2 9})\end{array}$ & $\mathbf{p}$ \\
\hline Ampolas & $56,1 \pm 13,5(36-86)$ & $47,3 \pm 10,6(24-72)$ & 0,017 \\
Foliculogênese (dias) & $12,1 \pm 2,2(8-17)$ & $11,3 \pm 1,6(8-14)$ & 0,156 \\
Folículos & $6,9 \pm 5,4(2-26)$ & $10,6 \pm 6,4(2-32)$ & 0,001 \\
Oócitos & $6,9 \pm 6,5(1-30)$ & $11,9 \pm 7,6(2-32)$ & 0,001 \\
Fertilização & $0,7 \pm 0,2(0,3-1)$ & $0,6 \pm 0,2(0,2-1)$ & 0,131
\end{tabular}

Resultados: média \pm desvio padrão

(variação)

\section{Discussão}

O tratamento mais apropriado para a endometriose associada à infertilidade é ainda objeto de controvérsia. Além disso, a conduta para os endometriomas é discutida e debatida, pelo fato de a taxa cumulativa de gravidez descrita após laparotomia ou laparoscopia para remoção ou cauterização de endometriose ovariana variar de
0 a $67 \%$ na literatura ${ }^{11,20,21}$, após 12 a 18 meses de seguimento.

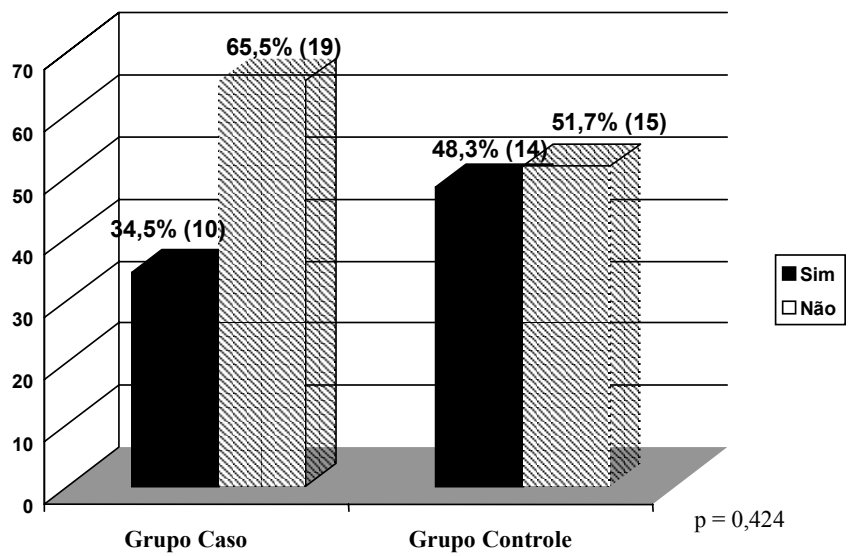

Figura 2 - Comparação entre as taxas de gestação após ciclos de reprodução assistida em pacientes $>35$ anos com cirurgia ovariana prévia e no grupo controle.

Alguns autores demonstraram que a cirurgia ovariana prévia para tratamento de endometrioma determina efeito deletério na resposta ovariana de pacientes submetidas a tratamento com FIV. Hornstein et al. ${ }^{14}$ demonstraram que pacientes com cirurgia ovariana prévia apresentavam $\mathrm{E}_{2}$ sérico mais baixo, menor número de folículos e maior taxa de cancelamento de ciclos de FIV usando citrato de clomifeno/hMG para indução da ovulação. Nargund et al. ${ }^{15}$ mostraram que a cistectomia prévia reduz o número de folículos $\mathrm{e}$ oócitos em ciclos de FIV usando GnRHa e HMG. Loh et al. ${ }^{16}$ observaram que a cistectomia prévia reduz o número de folículos em ciclos de FIV naturais e com citrato de clomifeno. Al-Azemi et al. ${ }^{22}$ mostraram redução na resposta ovariana e aumento no número de ampolas de FSH necessárias para a indução da superovulação em pacientes previamente submetidas a cirurgia ovariana. Tinkanen et al. ${ }^{23}$ observaram menor número de embriões e redução na taxa de gravidez em pacientes submetidas à cirurgia ovariana para tratamento de endometriomas quando comparadas a pacientes com endometrioma sem passado de cirurgia, em ciclos de FIV.

Nossos resultados demonstram que a cirurgia ovariana prévia reduz a resposta ovariana em ciclos de reprodução assistida. Mulheres com idade igual ou inferior a 35 anos e com cirurgia ovariana anterior apresentaram menor número de oócitos que o grupo controle. No grupo das mulheres com mais de 35 anos, observamos redução no número de folículos e oócitos, além da necessidade de maior número de ampolas para a indução da superovulação nas pacientes com cirurgia ovariana prévia, quando comparadas às do Grupo controle. 
Nós observamos que o impacto da cirurgia ovariana no ciclo de FIV foi maior nas pacientes com mais de 35 anos. Isso pode ser explicado pela própria redução da reserva folicular ovariana que é esperada a partir desta idade. Apesar de a taxa de gestação ter sido maior nas pacientes sem cirurgia ovariana anterior, os resultados obtidos não alcançaram significância estatística. Esse resultado pode ser explicado pelo limitado número de pacientes analisadas neste subgrupo.

A redução na resposta ovariana à indução da ovulação para FIV, em pacientes previamente submetidas a cirurgia ovariana, pode ser explicada pelo fato de a cirurgia remover fragmentos do córtex ovariano e diminuir a reserva folicular e, conseqüentemente, o número de folículos e oócitos obtidos após a indução. Além disso, a redução do fluxo sangüineo nas artérias ovarianas pode diminuir os niveis hormonais que atingem os ovários.

Yanushpolsky et al. ${ }^{24}$ sugeriram que os endometriomas podem produzir substâncias que são tóxicas para os oócitos, reduzindo, desse modo, sua qualidade e as taxas de fertilização e clivagem, além de promover aumento na taxa de perda gestacional precoce. Pal et al. ${ }^{25}$ sugeriram um impacto biológico adverso da endometriose grau III/IV sobre os oócitos, entretanto os resultados da FIV/ transferência de embriões não foram alterados. Esta conclusão está de acordo com o que foi observado por Geber et al. ${ }^{26}$, Olivennes et al. ${ }^{27}$ and Minguez et $a .^{28}$, que demonstraram que a presença e a gravidade da endometriose não afeta os resultados da FIV.

Nós não incluímos em nosso estudo um grupo de pacientes com endometriose, pois está bem demonstrado que a sua presença não interfere nos resultados da $\mathrm{FIV}^{28-30}$. Além disso, o objetivo do nosso estudo foi comparar apenas os efeitos da cirurgia ovariana prévia.

Podemos concluir que a cirurgia sobre os ovários, para o tratamento da endometriose, reduz a resposta ovariana durante ciclos de FIV/ ICSI em mulheres com mais de 35 anos, além de apresentar tendência a diminuir as taxas de gravidez. Desse modo, acreditamos que, para as pacientes inférteis com endometriose ovariana, o tratamento conservador deve ser considerado como primeira opção a fim de se evitar redução na resposta ovariana.

\section{ABSTRACT}

Purpose: to evaluate the effect of previous ovarian surgery for endometriosis on the ovarian response in assisted reproduction treatment cycles and its pregnancy outcome. Methods: a total of 61 women, with primary infertility and previous ovarian surgery for endometriosis, submitted to 74 in vitro fertilization/intracytoplasmic spermatozoid injection (IVF/ICSI) cycles, were studied (study group). A further 74 patients with primary infertility who underwent 77 IVF/ICSI cycles within the same period of time, at the same clinic and without previous ovarian surgery or endometriosis were studied as control group. Patients were matched for age and performed treatment. The groups were compared regarding number of ampoules used for superovulation, duration of folliculogenesis, number of follicles, number of oocytes, fertilization and pregnancy rate. IVF started with long protocol GnRHa for pituitary suppression followed by superovulation. After oocyte collection, in vitro insemination or sperm injection was performed and embryos were transferred from day 2 to day 5.

Results: patients $<35$ years with previous ovarian surgery had less oocytes retrieved than the patients of the control group $(p=0.049)$. Number of ampoules used for superovulation, duration of folliculogenesis, number of follicles, and fertilization rate were similar in both groups. The same was observed for pregnancy rates, as 24 patients (53.3\%) with previous ovarian surgery and $27(56.2 \%)$ of the control group became pregnant. Patients $>35$ years with previous ovarian surgery needed more ampoules for superovulation $(p=0.017)$ and had less follicles and oocytes than women of the control group ( $p=0.001)$. Duration of folliculogenesis was similar in both groups, as was fertilization rate. A total of 10 patients achieved pregnancy in the study group (34.5\%) and 14 $(48.3 \%)$ in the control group.

Conclusion: ovarian surgery for endometriosis reduced the ovarian outcome in IVF/ICSI cycles in women $>35$ years old, and might also decrease pregnancy rates. Therefore, we believe that for infertile patients, a conservative treatment might be a better option to avoid the reduction of ovarian response.

KEY WORDS: Infertility. Ovarian surgery. Endometriosis. In vitro fertilization.

\section{Referências}

1. Audebert A, Backstrom T, Barlow DH, et al. Endometriosis 1991: a discussion document. Hum Reprod 1992; 7:432-5.

2. Strathy JH, Molgaard CA, Coulam CB, Melton LJ 3rd. Endometriosis and infertility: a laparoscopic study of endometriosis among fertile and infertile women. Fertil Steril 1982; 38:667-72.

3. Balasch J, Creus M, Fabregues F, et al. Visible and non-visible endometriosis at laparoscopy in fertile and infertile women and in patients with chronic pelvic pain: a prospective study. Hum Reprod 1996; 11:387-91.

4. Mahmood TA, Templeton A. Prevalence and genesis of endometriosis. Hum Reprod 1991; 6:544-9. 
5. Pittaway DE, Maxson W, Daniell J, Herbert C, Wentz AC. Luteal phase defects in infertility patients with endometriosis. Fertil Steril 1983; 39:712-3.

6. Hirschowitz JS, Soler NG, Wortsman J. The galactorrhoea-endometriosis syndrome. Lancet 1978; 1:896-8.

7. Thomas EJ, Lenton EA, Cooke ID. Follicle growth patterns and endocrinological abnormalities in infertile women with minor degrees of endometriosis. Br J Obstet Gynaecol 1986; 93:852-8.

8. Kusuhara K. Luteal function in infertile patients with endometriosis. Am J Obstet Gynecol 1992; 167:274-7.

9. Senturk LM, Arici A. Immunology of endometriosis. J Reprod Immunol 1999; 43:67-83.

10.Sampson JA. Peritoneal endometriosis due to the menstrual dissemination of endometrial tissue into the peritoneal cavity. Am J Obstet Gynecol 1927; 14:422-69.

11.Adamson GD, Hurd SJ, Pasta DJ, Rodriguez BD. Laparoscopic endometriosis treatment: is it better? Fertil Steril 1993; 59:35-44.

12. Marcoux S, Maheux R, Berubé S. Laparoscopic surgery in infertile women with minimal or mild endometriosis. Canadian Collaborative Group on Endometriosis. N Engl J Med 1997; 337:217-22.

13.Diamond MP, Daniell JF, Feste J, et al. Adhesion reformation and de novo adhesion after reproductive pelvic surgery. Fertil Steril 1987; 47:864-6.

14. Hornstein MD, Barbieri RL, McShane PM. Effects of previous ovarian surgery on the follicular response to ovulation induction in an in vitro fertilization program. J Reprod Med 1989; 34:277-81.

15.Nargund G, Cheng WC, Parsons J. The impact of ovarian cystectomy on ovarian response to stimulation during in-vitro fertilization cycles. Hum Reprod 1996; 11:81-3.

16.Loh FH, Tan AT, Kumar J, Ng SC. Ovarian response after laparoscopic ovarian cystectomy for endometriotic cysts in 132 monitored cycles. Fertil Steril 1999; 72:316-21.

17.Hemmings R, Bissonnette F, Bouzayen R. Results of laparoscopic treatments of ovarian endometriomas: laparoscopic ovarian fenestration and coagulation. Fertil Steril 1998; 70:527-9.
18.Geber S, Sampaio M. Blastomere development after embryo biopsy: a new model to predict embryo development and to select for transfer. Hum Reprod $1999 ; 14: 782-6$.

19.Sampaio MA, Geber S. Births after transfer of zonafree blastocysts in oocyte donation cycles. J Assist Reprod Genet 2001; 18:156-9.

20.Nezhat C, Crowgey S, Nezhat F. Videolaseroscopy for the treatment of endometriosis associated with infertility. Fertil Steril 1989; 51:237-40.

21. Murphy AA, Schlaff WD, Hassiakos D, Durmusoglu F, Damewood MD, Rock JA. Laparoscopic cautery in the treatment of endometriosis related infertility. Fertil Steril 1991; 55:246-51.

22.Al-Azemi M, Bernal AL, Steele J, Gramsbergen I, Barlow D, Kennedy S. Ovarian response to repeated controlled stimulation in in-vitro fertilization cycles in patients with ovarian endometriosis. Hum Reprod 2000; 15:72-5.

23.Tinkanen $\mathrm{H}$, Kujansuu E. In vitro fertilization in patients with ovarian endometriomas. Acta Obstet Gynecol Scand 2000; 79:119-22.

24.Yanushpolsky EH, Best CL, Jackson KV, Clarke RN, Barbieri RL, Hornstein MD. Effects of endometriomas on oocyte quality, embryo quality, and pregnancy rates in in vitro fertilization cycles: a prospective, case-controlled study. J Assist Reprod Genet 1998; 15:193-7.

25.Pal L, Shifren JL, Isaacson KB, Chang Y, Leykin L, Toth TL. Impact of varying stages of endometriosis on the outcome of in vitro fertilization-embryo transfer. J Assist Reprod Genet 1998; 15:27-31.

26.Geber S, Paraschos T, Atkinson G, Margara R, Winston RM. Results of IVF in patients with endometriosis: the severity of the disease does not affect the outcome, or the incidence of miscarriage. Hum Reprod 1995; 10:1507-11.

27. Olivennes F, Feldberg D, Liu HC, Cohen J, Moy F, Rosenwaks Z. Endometriosis: a stage by analysis the role of in vitro fertilization. Fertil Steril 1995; 64:392-8.

28. Minguez Y, Rubio C, Bernal A, et al. The impact of endometriosis in couples undergoing intracytoplasmic sperm injection because of male infertility. Hum Reprod 1997; 12:2282-5. 